\title{
Effect of culture operating conditions on succinate production in a multiphase fed-batch bioreactor using an engineered Escherichia coli strain
}

\author{
Jiangfeng Zhu • Chandresh Thakker • Ka-Yiu San • \\ George Bennett
}

Received: 13 March 2011 /Revised: 11 April 2011 /Accepted: 13 April 2011 /Published online: 11 June 2011

(C) Springer-Verlag 2011

\begin{abstract}
A metabolically engineered Escherichia coli strain SBS550MG (pHL413) was used in this study to investigate the impact of various culture operating conditions for improving the specific succinate production rate for better final titer while maintaining the theoretical succinate yield on glucose in multiphase fed-batch cultures. Previously, we reported that changes in the level of aeration during the cell growth phase significantly modified gene expression profiles and metabolic fluxes in this system (Martinez et al. 2010). Based on these observations, the examination of culture conditions was mainly focused on the aerobic growth phase. It was found that $2-5 \mathrm{~h}$ of low dissolved oxygen culture during the aerobic phase improves cell productivity, but $\mathrm{pH}$ control during the aerobic phase
\end{abstract}

\section{K.-Y. San}

Department of Bioengineering, Rice University,

Houston, USA

C. Thakker $\cdot$ G. Bennett $(\bowtie)$

Department of Biochemistry and Cell Biology, MS-140,

Rice University,

6100 Main Street,

Houston TX77005-1892, USA

e-mail: gbennett@rice.edu

\section{K.-Y. San}

Department of Chemical and Biomolecular Engineering,

Rice University,

Houston, USA

\section{J. Zhu}

Qingdao Institute of Bioenergy and Bioprocess Technology, Chinese Academy of Sciences,

Qingdao, China

J. Zhu

Key Laboratory of Biofuels, Chinese Academy of Sciences, Qingdao, China was not favorable for the system. Cell viability has been identified as a major limiting factor for succinate production. Supplementing LB medium and betaine, an antiosmotic stress reagent, did not improve cell activity. A higher succinate titer $(537.8 \mathrm{mM})$ using the current metabolic engineering $E$. coli strain was achieved, which can potentially be improved further by increasing cell viability.

Keywords Succinate production $\cdot$ Escherichia coli $\cdot$ Culture conditions $\cdot$ Multiphase fermentation

\section{Introduction}

Chemical production using bioprocesses are becoming increasingly competitive as environmental concerns and fossil raw material cost increases. The US Department of Energy has identified 12 sugar-derived chemicals as top value-added chemicals from biomass, and, among them, succinic acid was considered as one of the most promising chemicals (Werpy and Petersen 2004). Succinic acid is an intermediate of the tricarboxylic acid cycle and one of the fermentation end-products of anaerobic metabolism. It can serve as a precursor of numerous commercial chemicals including pharmaceuticals and biodegradable polymers (Hong and Lee 2002).

There is a significant market opportunity for the development of biobased industrial production of succinic acid. In the past few years, some natural microbial strains such as Actinobacillus succinogenes, Anaerobiospirillum succiniproducens, and Mannheima succiniproducens were found to be able to produce succinate as a major metabolite along with ethanol, acetate and lactate as cometabolites (Lee et al. 2003, 2001, 2006; McKinlay et al. 2005; Oh et 
al. 2008). Since the cultures of these natural succinate producing strains are more difficult to operate as compared with the popular genetic engineering strains, a variety of metabolic engineered Escherichia coli strains (Jantama et al. 2008; Sanchez et al. 2005; Vemuri et al. 2002) and Saccharamyces cerevisiae strains (Otero et al. 2007) were developed for succinic acid bioproduction. The succinate yields in these $E$. coli systems were reported to be between 1.16 and $1.7 \mathrm{~mol} / \mathrm{mol}$ glucose in the cultures. The major challenges to make the succinate bioproduction economically viable are primarily associated with reducing the overall cost of the fermentation. Three main issues, such as using a low cost medium, a high succinate yield on the carbon source, and volumetric productivity above $2.5 \mathrm{~g} \mathrm{~L}^{-1} \mathrm{~h}^{-1}$, must be addressed so that the biobased succinate production can be commercialized (Werpy and Petersen 2004).

The strain used in this work was SBS550MG (pHL413), created by Sánchez and et al. (2005). This strain has the following genes deleted, adhE, ldhA, iclR, and ackA-pta and overexpresses the gene $p y c$ encoding for pyruvate carboxylase from Lactococcus lactis. This strain has shown to produce succinate at high yield, $1.6 \mathrm{~mol}$ succinate $/ \mathrm{mol}$ glucose, in a dual-phase system. The first phase was aerobic and the cells were grown in shake flasks followed by an anaerobic phase where succinate was produced in shake flasks or batch bioreactor (Sanchez et al. 2005, 2006). This yield value is also the theoretical maximum for this strain (Cox et al. 2006 and Sanchez et al. 2006). Recently, Martinez et al. (2010) have reported the metabolic effect of two different aeration conditions during the growth phase of SBS550MG (pHL413) on metabolite and gene expression profiles, as well as the metabolic fluxes, during the production phase. The metabolic flux distributions and product yields during the anaerobic phase were significantly affected by the levels of aeration during the growth phase. The low aeration experiment showed better succinate production with respect to yield, titer, and volumetric productivity (Martinez et al. 2010).

The metabolic behavior of a cell is highly influenced by the operation conditions of the process. Appropriate process conditions are crucial for the metabolic engineered strains to reach their maximum potential, as they may allow the modified metabolic routes to behave as predicted. The physiological state of the cells is critical in a dual-phase system where the enzymes produced during the first phase (aerobic-growth phase) will influence the performance of the cells in the anaerobic-production phase. In the present study, we examined the influence of growth phase operating conditions, such as dissolved oxygen (DO), culture temperature, additional nutrient feeding, and $\mathrm{pH}$ control on succinate production during the anaerobic phase using E. coli strain SBS550MG (pHL413) in a bioreactor.
The objective is to improve the strain performance for better succinate production rates and increasing the final titer while maintaining the theoretical succinate yield on glucose.

\section{Materials and methods}

Microorganism and culture conditions

The succinate-producing E. coli strain SBS550MG (pHL413) was previously constructed from MG1655 wild type. The mutant strain lacks the $i c l \mathrm{R}, a d h \mathrm{E}, l d h \mathrm{~A}$, and ackA-pta genes, while bearing a plasmid expressing the pyc gene from L. lactis, which encodes the pyruvate carboxylase enzyme for $\mathrm{CO}_{2}$ fixation (Sanchez et al. 2005).

The culture medium contains $(1 \mathrm{~L}): 20 \mathrm{~g}$ of tryptone, $10 \mathrm{~g}$ of yeast extract, $2.4 \mathrm{mmol}$ of $\mathrm{K}_{2} \mathrm{HPO}_{4}, 5.1 \mathrm{mmol}$ of $\mathrm{KH}_{2} \mathrm{PO}_{4}$, and $13.8 \mathrm{mmol}$ of $\left(\mathrm{NH}_{4}\right)_{2} \mathrm{SO}_{4}$. A small amount of antifoam reagent 204 (Sigma) was added to the medium $(30 \mu \mathrm{l} / \mathrm{L})$ and the medium $\mathrm{pH}$ was adjusted to 7.5 before autoclaving. The solutions of $1 \mathrm{mmol} \mathrm{CaCl}_{2}, 1.2 \mathrm{mmol}$ $\mathrm{MgSO}_{4}, 0.6 \mathrm{mg}$ of thiamine, and $2 \mathrm{~g}$ of glucose were filter sterilized separately and added to the medium after autoclaving.

A 1-L bioreactor (BioFlo 110, New Brunswick Scientific, Edison, NJ, USA) was used for the cultures and the initial working volume was kept at $600-800 \mathrm{ml}$ depending on the DO conditions. The temperature was maintained at $37{ }^{\circ} \mathrm{C}$ unless otherwise mentioned. The fed-batch cultures were started with aerobic cultures with the agitation speed controlled between 500 and $800 \mathrm{rpm}$ depending on the DO conditions and glucose was fed into the medium in a batch-wise mode so that the culture $\mathrm{pH}$ would not exceed the range between 6.5 and 7.5. The reaction was purged with air at a flow rate of $1-1.5 \mathrm{~L} /$ min during the aerobic phase and the purge gas was changed into $\mathrm{CO}_{2}$ at a flow rate of $0.2 \mathrm{~L} / \mathrm{min}$ when switching into anaerobic culture conditions. The agitation speed was changed to $250 \mathrm{rpm}$ when switching to anaerobic conditions. A batch of glucose was fed into the culture when switching to anaerobic phase so that the glucose concentration in the culture will be about 100 $150 \mathrm{mM}$. About $4.0 \mathrm{~g}$ of $\mathrm{MgCO}_{3}$ suspension in sterile water was also fed into the bioreactor to control the $\mathrm{pH}$.

Analytical procedures

Cell growth was monitored by optical density measurements at $600 \mathrm{~nm}$ using a UV/visible spectrophotometer (Spectronic 1001, Bausch and Lomb). Samples were diluted appropriately with $0.9 \% \mathrm{NaCl}$ to keep the $\mathrm{OD}_{600}$ value between 0.1 and 0.5 . 
To measure the extracellular metabolite concentrations, $1 \mathrm{ml}$ of sample was centrifuged and then the supernatant was filtered through a $0.45-\mu \mathrm{m}$ pore size filter for analysis by high-performance liquid chromatography (HPLC). The HPLC system (Shimadzu-10A Systems, Shimadzu, Columbia, MD, USA) was equipped with a cation-exchange column (Aminex HPX-87 H, BioRad Labs, Hercules, CA, USA), a UV detector (Shimadzu SPD-10A), and a differential refractive index detector (Waters 2410, Waters, Milford, MA, USA). The mobile phase was $2.5 \mathrm{mM} \mathrm{H}_{2} \mathrm{SO}_{4}$ at a steady flow rate $0.5 \mathrm{ml} / \mathrm{min}$. The column temperature was maintained at $55{ }^{\circ} \mathrm{C}$ (Sanchez et al. 2005; Yang et al. 1999).

\section{Enzyme assay}

Cell extracts of the E. coli strain which is equivalent to $100 \mathrm{OD}_{600}$ units were prepared by washing the cell pellets with phosphate buffered saline (PBS) buffer (pH 7.4) and the cell wall was broken by 10 sonication steps in the ultrasonic disrupter with 30 -s intervals between each step. Cell debris was removed by centrifugation $(10,000 \times g$ for $30 \mathrm{~min}$ at $4{ }^{\circ} \mathrm{C}$ ), and cell extracts were used to measure enzyme activities. The enzyme activity of pyruvate carboxylase (Pyc) was measured in a $1 \mathrm{ml}$ mixture in PBS buffer (pH 7.4) containing $0.05 \mathrm{M} \mathrm{NaHCO}_{3}, 0.05 \mathrm{M} \mathrm{MgCl}_{2}$, $5 \mathrm{mM}$ AcCoA, $5 \mathrm{mM}$ ATP, $0.1 \mathrm{~g} / \mathrm{L}$ DNTB, $10 \mathrm{mM}$ pyruvate, and five units of citrate synthase. The mixture was incubated at $37{ }^{\circ} \mathrm{C}$ and the absorbance changes at $412 \mathrm{~nm}$ were measured using a spectrophotometer (Thermo Fischer UNICAM UV1). The total protein in the cell extracts was determined with Bradford's reagent (Sigma) and bovine serum albumin as the standard.

\section{Cell viability measurement using flow cytometry}

Cell viability was measured using cell pellets collected by spinning down $1 \mathrm{ml}$ of culture broth followed by washing the cells twice with PBS buffer ( $\mathrm{pH}$ 7.4). Then the cell pellets were resuspended in PBS buffer to a final $\mathrm{OD}_{600}$ value between 0.01 and 0.05 . As propidium iodide (PI, Sigma) can traverse the membrane of cells with compromised membranes and bind to the DNA (fluorescing red), the cells were stained with PI at a concentration of $2 \mathrm{mg} / \mathrm{L}$ and left at room temperature for $5 \mathrm{~min}$ to identify dead or injured cells (Portle et al. 2007). The flow cytometer used was a FACScalibur (BD Biosciences), with a $15 \mathrm{~mW}, 488 \mathrm{~nm}$, air-cooled argon-ion laser. All parameters were logarithmically amplified, with the following settings: FSC E01, SSC $381 \mathrm{~V}$, FL1 $601 \mathrm{~V}$, FL2 $500 \mathrm{~V}$, FL3 $575 \mathrm{~V}$. A side scatter threshold was applied to gate out much of the noise (at channel 130). Twenty thousand to 40,000 events were collected for each sample. The flow cytometer was calibrated with EGFP calibration beads (Clonetech).

\section{Results}

Batch culture phase shifts

The cell growth and metabolite production were significantly different as the culture phase shifted. As shown in Fig. 1, a typical batch culture for succinate production was started with $2 \mathrm{~g} / \mathrm{L}$ of glucose under aerobic conditions using a $1-1.5 \mathrm{~L} / \mathrm{min}$ of air purge and a $500-800 \mathrm{rpm}$ agitation speed. The $\mathrm{pH}$ was not controlled during the aerobic phases. During the phase A-I, the $\mathrm{pH}$ decreased as glucose was consumed and then rose again as the organic acids produced by glucose metabolism were utilized by the cells in the absence of glucose. After the phase A-I, at least one batch of $2 \mathrm{~g} / \mathrm{L}$ glucose was fed into the system. During the phase A-II, DO in the culture system was always at a relatively low level $(0-20 \%)$. The length of phase A-II was controlled by glucose feeding. Before switching to the anaerobic phase AN, samples were taken and analyzed by HPLC which showed almost no organic acid in the system (data not shown). When switching to the phase AN, the purging gas was switched to $\mathrm{CO}_{2}$ at a slow but steady rate $(0.2 \mathrm{~L} / \mathrm{min})$, while the agitation speed was slowed down to $250 \mathrm{rpm}$. At the beginning of the phase AN, $20 \mathrm{~g} / \mathrm{L}$ of glucose was feed into the system as carbon source for succinate production and $\mathrm{pH}$ control was turned on to control the $\mathrm{pH}$ at about 7.0 using $\mathrm{NH}_{4} \mathrm{OH}$ or $\mathrm{NaOH}$.

In this typical bioreactor experiment and some other trials, we observed that the succinate production rate dropped quickly after about $20 \mathrm{~h}$ in the production phase. Therefore, cell viability was checked by PI staining using the flow cytometer. As shown in Fig. 2a, c, a larger population of cells seems to extend their sizes after longer production phase. More significantly, the PI staining samples showed a bigger peak in the $23.5 \mathrm{~h}$ sample compared to the 14-h sample during the AN phase, which indicates that larger portion of cells were damaged (Fig. 2b, d).

Effect of air supply conditions in the aerobic phases

The air supply conditions were mainly controlled by changing the agitation speed during the phases A-I and AII. The 10 experiments used to compare the effect of air supply have three agitation setting patterns namely $800 \mathrm{rpm}$ in both phases A-I and A-II $(800+800), 800 \mathrm{rpm}$ in phase A-I and $500 \mathrm{rpm}$ in phase A-II $(800+500)$, and $500 \mathrm{rpm}$ in both phases A-I and A-II $(500+500$; Fig. 3 b). The overall molar succinate yields on glucose during the production 
Fig. 1 Bioreactor culture phases of a typical succinate fed-batch culture. The basic culture conditions and engineered strain are described in Methods

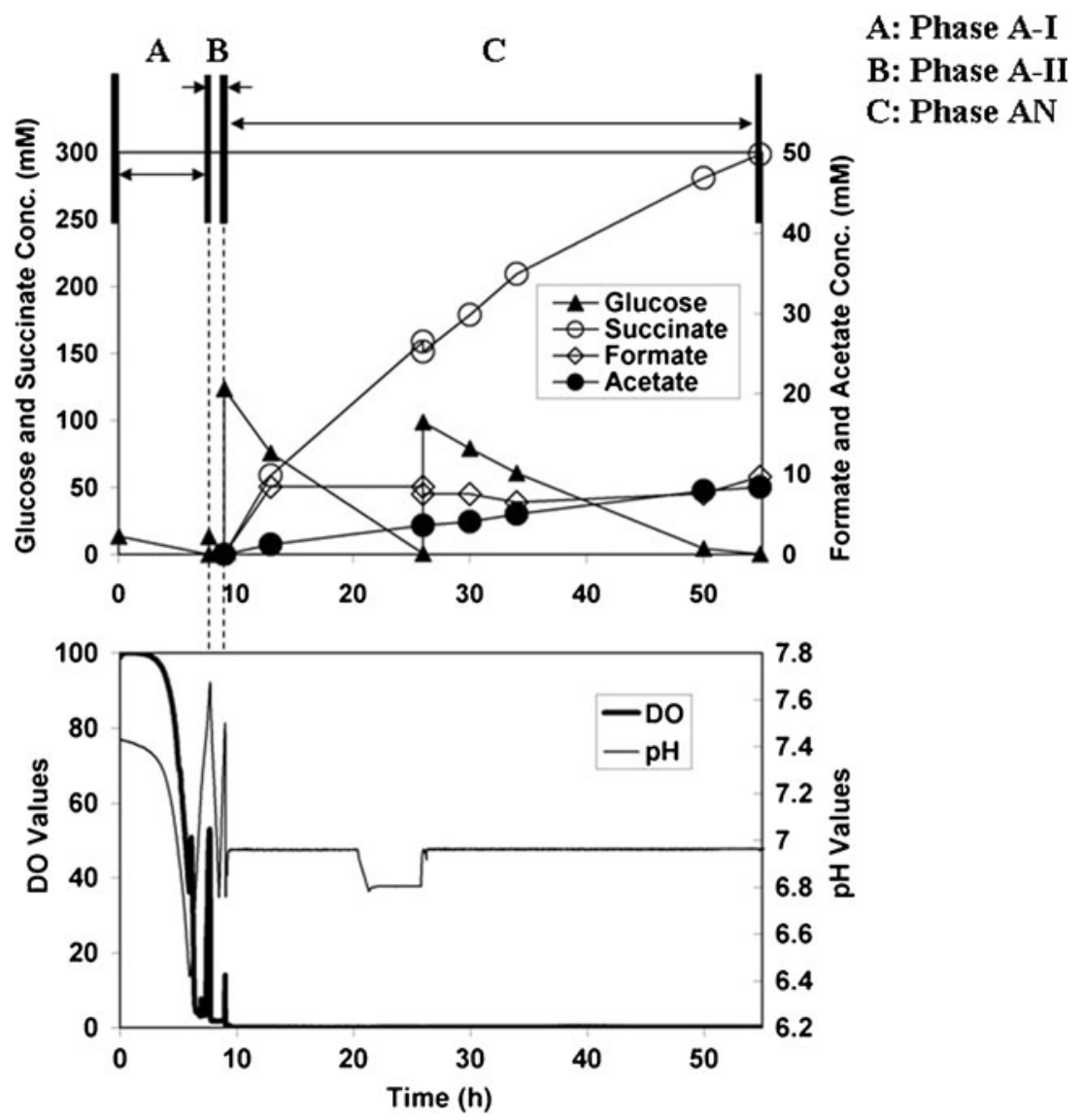

phase AN vary from 1.24 to 1.60 in these batch cultures (Fig. 3a). The specific succinate production rates vary from 0.08 to $0.12 \mathrm{~g} / \mathrm{L} / \mathrm{h} / \mathrm{OD}$. While high-agitation speed $(800+$ 800 and $800+500)$ runs gave relatively good succinate yields on glucose, the relatively lower agitation speed runs were more reproducible and resulted in about 1.56$1.60 \mathrm{~mol} / \mathrm{mol}$ succinate yields on glucose, and the specific production rate was not much lower than higher agitation speed setups. Therefore, the stable condition $(500+500)$ was used for further bioreactor cultures.

We noticed that the length of phase A-II (low DO incubation period) has some impact on the strain's performance during the production phase (AN). Therefore, a series of batch cultures were setup to evaluate the effect of the length of the low DO incubation period. As shown in Fig. 4, the A-II phase time length varies between 0.5 and $7 \mathrm{~h}$. The overall succinate yields in these batches were not significantly affected. However, a short low DO incubation period seems to favor a higher specific succinate production rate. In the two cultures where the A-II phase was about $7 \mathrm{~h}$, the specific succinate production rates were significantly lower, which resulted in lower overall production rate and thus lower final succinate titer. The optimal A-II phase time length seems to be between $2-5 \mathrm{~h}$.
Since a too long low-DO incubation time resulted in less succinate production, we wondered if that was due to the low cell viability or low metabolic activities in these cultures. Providing more air may help cells to keep a high metabolic activity so it may help to improve the production phase performance. With that in mind, we examined a culture where we controlled DO at about $8 \%$ in the A-II phase instead of allowing the DO to be less than $5 \%$ as in normal batch cultures. The initial glucose feeding during the switch to the production phase was increased to about $40 \mathrm{~g} / \mathrm{L}$ instead of $20 \mathrm{~g} / \mathrm{L}$ to avoid repeated osmotic stress shock when feeding glucose during the AN phase. However, the succinate yield and the initial specific production rate of the culture were similar to the previous runs, and the final succinate concentration was not improved (data not shown).

\section{Effect of additional nutrients feeding}

We noticed that the succinate production rate decreases with time after switching to the anaerobic phase. In addition, when the low DO incubation time was too long $(5-7 \mathrm{~h})$, the cell performance was not as good as those incubated at low DO for shorter time periods. These 
Fig. 2 Cell viability comparisons at two different time points during phase $\mathrm{AN}$. a Cell size population distribution of $\mathrm{AN}$ 14-h sample. b Red fluorescent detection for the AN-14-

h sample after PI staining. c Cell size population distribution of AN-23.5-h sample. d Red fluorescent detection for the AN23.5-h sample after PI staining. The two light scattering properties, forward, and side scatter (FSC and SSC, respectively) are measures of cellular size and internal complexity, respectively. FL3-H represents the relative red fluorescence strength. M1 in b and $\mathbf{d}$ marked out the PI stained peaks, which indicates the injured or dead cells

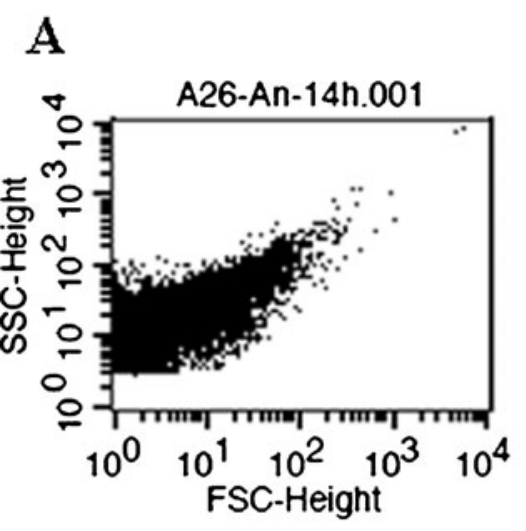

B
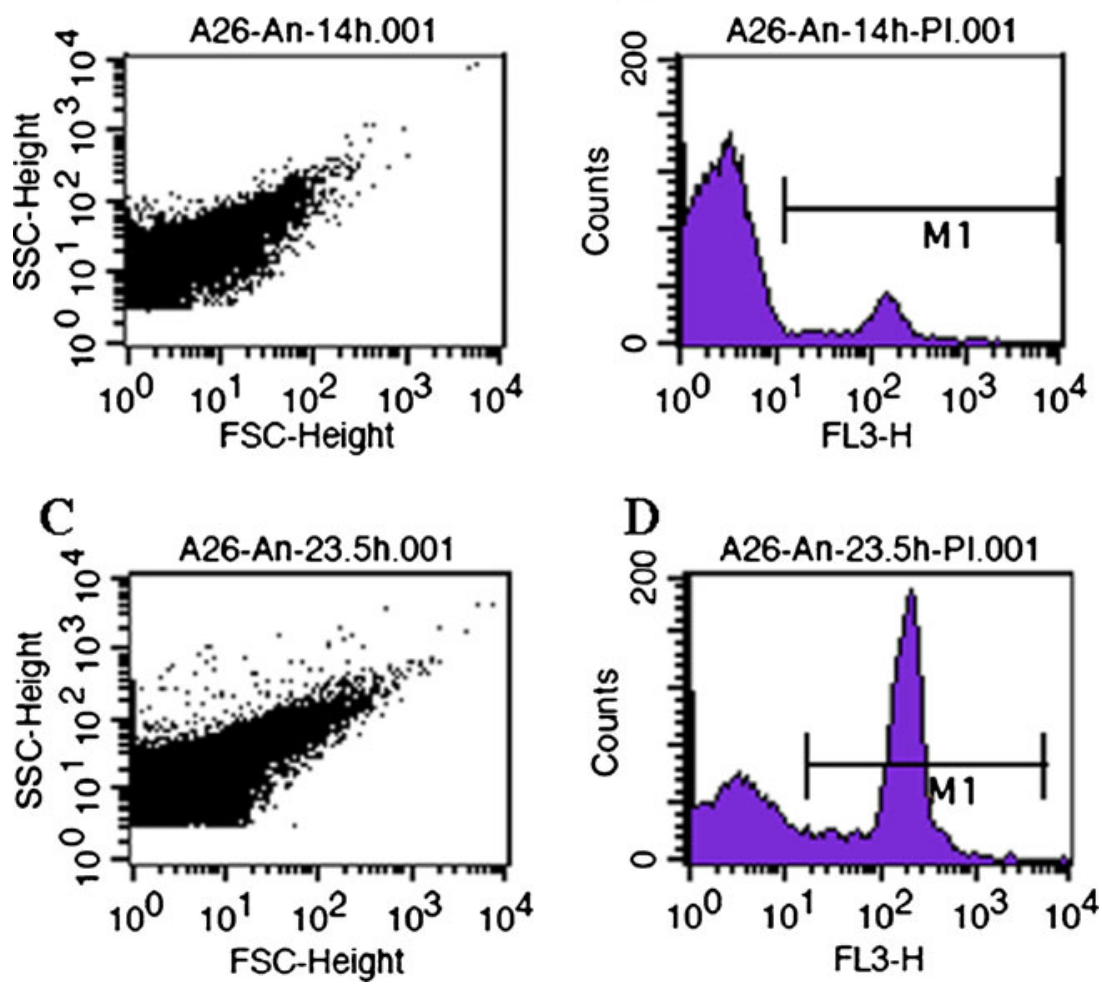

observations suggest that there might be some nutrient or energy limitation in the cultures.

To supplement nutrients so that cell can grow better and have better viability while maintaining succinate producing capacity, concentrated luria bertani broth (LB) medium (10 $\mathrm{ml}$ of $20 \times \mathrm{LB}$ each time) was fed into the culture medium during the phase A-II and at the switch to the production phase AN. As shown in Fig. 5, the batch cultures with no LB supplementation (Control), one LB supplementation during the A-II phase $[\mathrm{AII}(1 \times)]$, five LB supplementations during the A-II phase $[\mathrm{AII}(5 \times)]$, and three LB supplementations during the anaerobic phase $[\mathrm{AN}(3 \times)]$ were compared for their specific succinate production rates, succinate yields on glucose, and final succinate concentrations. The final succinate titer was slightly higher in the two cultures $[\mathrm{AII}(1 \times)$ and $\mathrm{AII}(5 \times)]$ with LB supplementations during the aerobic phase, as the initial cell concentrations and initial specific succinate production rates were both relatively higher in these two cultures compared with the culture without LB supplementation. As compared to culture without LB supplementation, the succinate yield on glucose was not significantly affected in LB supplemented cultures. The LB supplementation during the anaerobic phase did not improve cell performance as observed by low specific succinate production rate (Fig. 5).

As another factor, it is considered that the cell may experience significant osmotic stress as succinate production goes on during the AN phase. Therefore, we added
$1 \mathrm{mM}$ of betaine into the culture medium at the beginning of the aerobic phase (Fig. 5 (AN-BT)). However, succinate production was not improved in the presence of betaine (Fig. 5).

Previously, it was reported that the activities of glyoxylate shunt enzymes are important for metabolic cofactor balance in the current succinate production system (Sanchez et al. 2005) and it is known that the glyoxylate shunt is activated while using acetate as a carbon source due to the phosphorylation regulation of the isocitrate dehydrogenase (Cozzone 1998; Walsh and Koshland 1985). We supplemented $10 \mathrm{mM}$ of acetate at the end of the phase A-II in order to further activate the glyoxylate shunt so that the metabolic network can be further fine tuned. However, the succinate production in the AN phase was not improved (data not shown).

\section{Effect of $\mathrm{pH}$ control during aerobic phase}

Several $\mathrm{pH}$ control strategies during the aerobic phase were tested. The medium $\mathrm{pH}$ was controlled at 6.2 [A6 (pH 6.2)], 6.3 [A8 (pH 6.3)], and 7.0 [A17 (pH 7.0)], respectively, using $\mathrm{NaOH}$ and $\mathrm{HNO}_{3}$ during the aerobic phase. As a control, another culture without $\mathrm{pH}$ control during the aerobic phase [A48 (no control)] was used to compare with the aforementioned ones. As shown in Fig. 6, all four batch cultures with $\mathrm{pH}$ control resulted in low molar succinate yield on glucose ranging from 0.56 to 1.30 , and the initial 
A

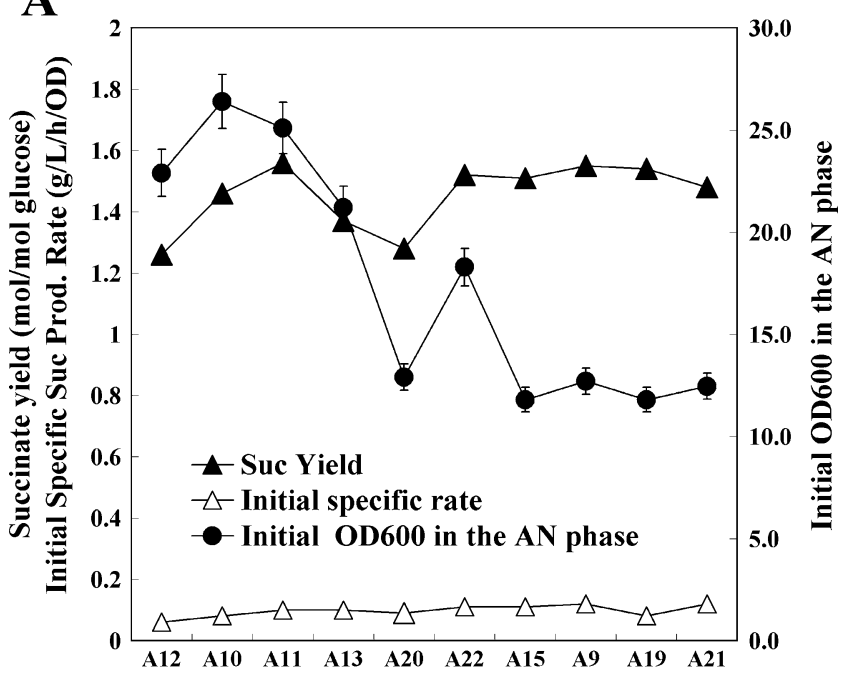

B

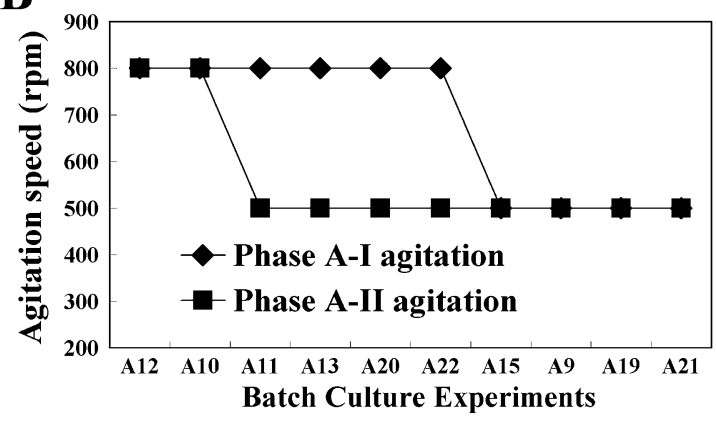

Fig. 3 Effect of DO settings in the aerobic phases. a Succinate yield and initial specific succinate production rate; $\mathbf{b}$ agitation speeds in the A-I and A-II phases

specific succinate production rate was significantly lower than that of the culture without $\mathrm{pH}$ control during the aerobic phase.

Effect of culture temperature in the aerobic phases

The expression of the heterologous pyruvate carboxylase gene $p y c$ was considered to be a rate-limiting factor in this succinate-producing system (Sanchez et al. 2005; Martinez et al. 2010). The pyc gene size is relatively large (about $3.4 \mathrm{~kb}$ ) and the recombinant expression of heterologous proteins in $E$. coli often results in protein aggregation (Baneyx 1999; Haacke et al. 2009). To reduce the protein synthesis rate to potentially improve the Pyc protein expression, several lower temperature cultures were tested for succinate production. As shown in Fig. 7, cell growth was not significantly affected by the temperature difference. All four cultures produced cells equivalent to $16 \mathrm{OD}_{600}$ units before switching to the production phase. However, the specific succinate production rate, succinate yield, and the final succinate titer were significantly increased as the
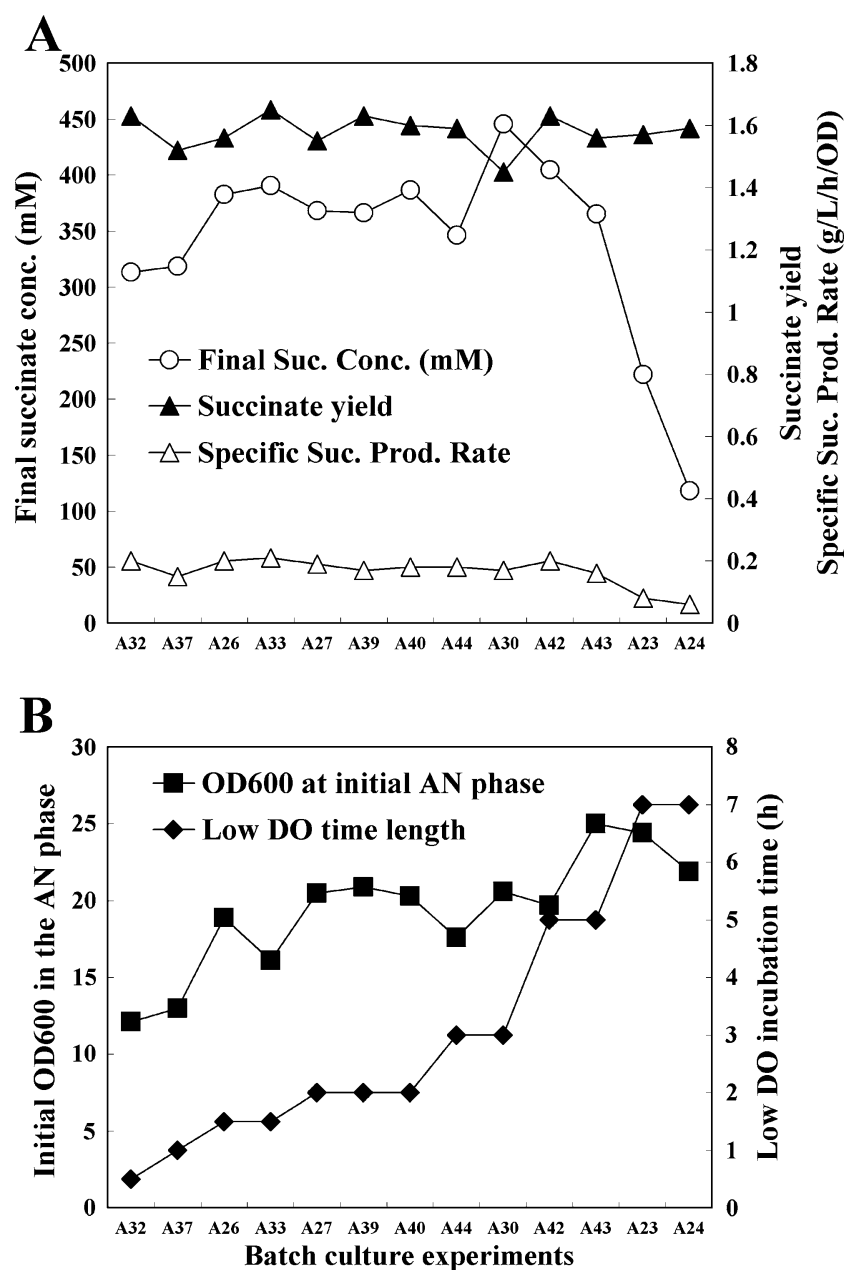

Fig. 4 Effect of low DO incubation time lengths during phase A-II. a Succinate yield, initial specific succinate production rate, and final succinate titer; b Low DO time length during A-II phase and initial cell density in the AN phase

aerobic culture temperature increased from 27 to $37^{\circ} \mathrm{C}$. We measured the Pyc activities when switching to the anaerobic phase in these cultures. As shown in Fig. 7, it seems that the lower temperature did not improve Pyc protein expression. On the other hand, the lower culture temperature still resulted in reasonable Pyc activity as compared to the culture at $37^{\circ} \mathrm{C}$.

High cell density culture

A high cell density fed-batch culture was examined for the final succinate titer using the suitable culture and medium conditions. As shown in Fig. 8, with four batches of glucose feeding, the final succinate concentration reached about $537.8 \mathrm{mM}$, along with $24 \mathrm{mM}$ of formate and $52 \mathrm{mM}$ of acetate as side products. It can be seen that the succinate production rate was faster in the first $10 \mathrm{~h}$ compared to the later production phase and the formate accumulation increased significantly after the fourth glucose feeding. 


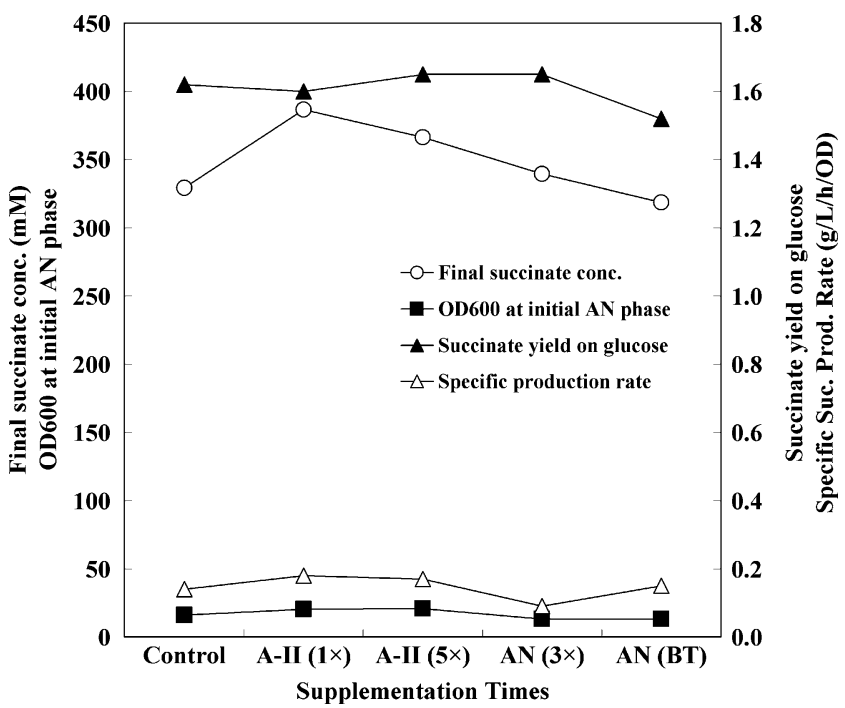

Fig. 5 Effect of LB medium supplementation. The $x$-axis denotes the batch cultures with no LB supplementation (control), one LB supplementation during the A-II phase [AII $(1 \times)]$, five LB supplementations during the A-II phase $[\mathrm{AII}(5 \times)]$, three LB supplementations during the anaerobic phase $[\mathrm{AN}(3 \times)]$ and $1 \mathrm{mM}$ of betaine at the beginning of the anaerobic phase [AN(BT)]

The concentration was reduced due to the dilution effect, which indicates the succinate production stopped after about $25 \mathrm{~h}$.

\section{Discussion}

The operation conditions of the process greatly affect the metabolic behavior of the production strain. The genetic manipulation themselves are not enough to make a strain a high producer or achieve maximum theoretical yield. Key process conditions are important for the engineered strains to reach their maximum potential as they may allow the modified metabolic routes to behave as predicted. In the present study, we studied the influence of crucial growth

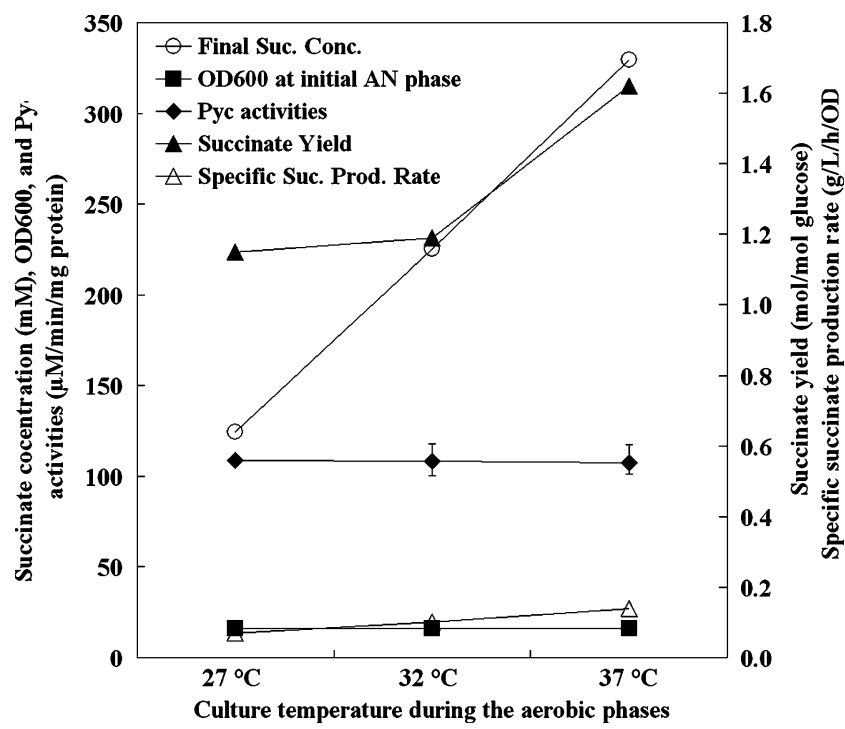

Fig. 7 Effect of aerobic phase culture temperatures. The basic culture conditions are as in Fig. 1 with the aerobic phase temperature adjusted as indicated

phase operating conditions, such as DO, additional nutrient feeding, $\mathrm{pH}$ control, and culture temperature on succinate production using E. coli strain SBS550MG (pHL413) in a multiphase bioreactor process. The first phase A-I was aerobic for biomass accumulation followed by second phase A-II of low DO and third phase of anaerobic succinate production. The metabolic flux distribution in the central metabolic pathways and cell viability are two important factors can affect the final succinate production rate, yield and final titer, and the metabolic flux is determined by the genetic background and operating conditions such as air supply, $\mathrm{pH}$ control, culture temperature, etc.

The air supply conditions in the aerobic phases A-I and A-II showed significant effects on the succinate production in the anaerobic phase AN (Fig. 3). On one hand, the air supply conditions in phases A-I and A-II affect the cell
Fig. 6 Effect of $\mathrm{pH}$ control during the aerobic phase. The basic culture conditions are as in Fig. 1 with the aerobic phase $\mathrm{pH}$ adjusted as indicated

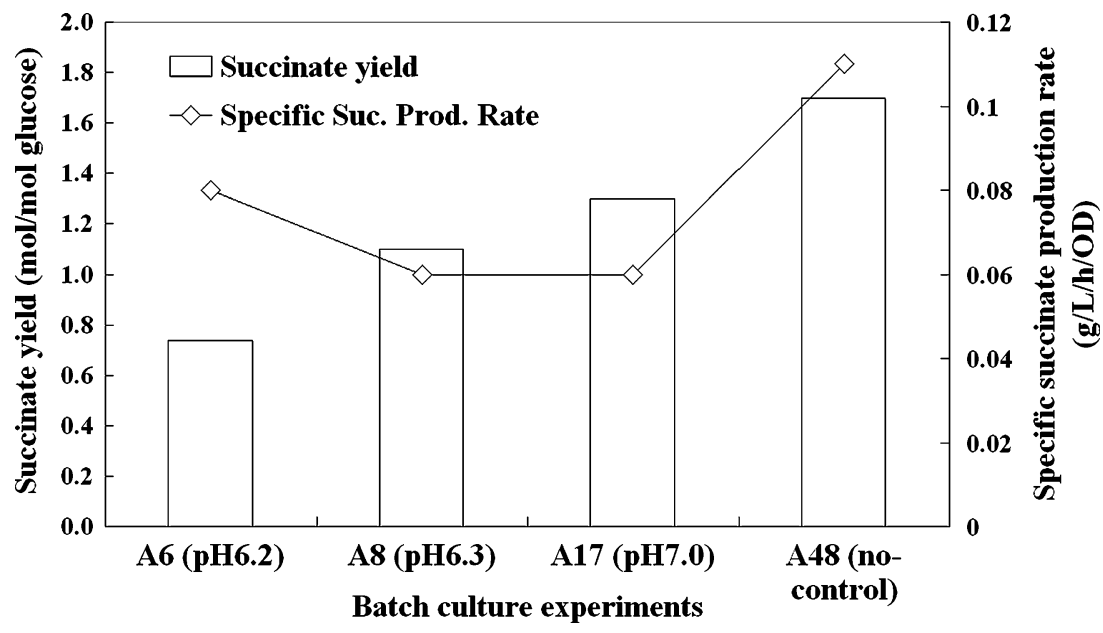




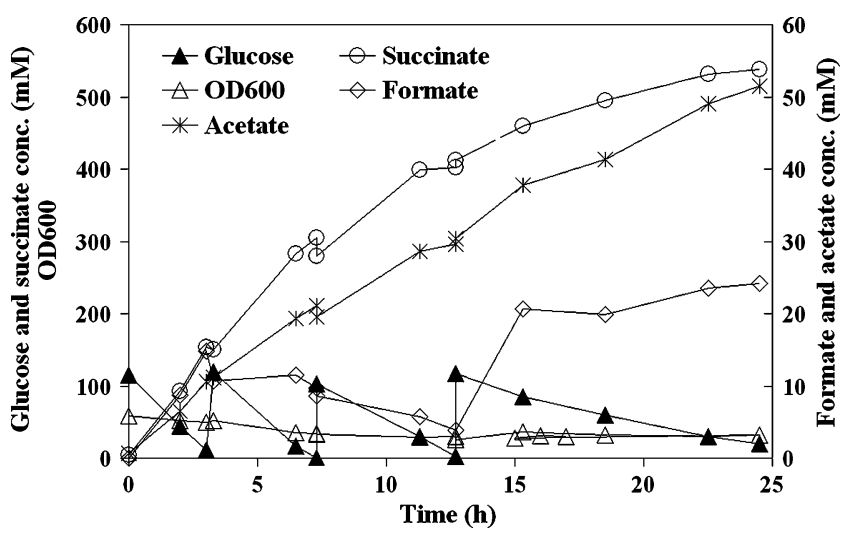

Fig. 8 High cell density batch culture for succinate production. The culture conditions are described in "Materials and methods"

density and cell viability at the switch to phase AN, which affects the overall production rate and the final succinate titer. On the other hand, the air supply conditions also affected the pathway fine-tuning in the cell, which resulted in variant specific succinate production rates and succinate yields on glucose.

Employing higher agitation speed improves biomass yield during the aerobic phase (Fig. 3). However, succinate productivity was not stable when using $800 \mathrm{rpm}$ during the $\mathrm{AI}$ and A-II phases. Besides, pyruvate accumulation was observed in high DO experiments. A recent extensive investigation indicated that appropriate aeration level during the aerobic phase changed the expression of the pfl $\mathrm{AB}$, ace $\mathrm{AB}$, and acn $\mathrm{AB}$ genes (Martinez et al. 2010). The elevated expression levels of these genes facilitate pyruvate conversion through pyruvate formate lyase and the glyoxylate shunt during the anaerobic phase. Together with the heterologous pyruvate carboxylase, this shunt balances reducing power usage and carbon processing at the pyruvate node, which resulted in good succinate yield (Cox et al. 2006).

Consistently, the low DO incubation time not only fine tunes the balance between the Pyc and the Pfl-glyoxylate shunt, which are important for succinate yield, since the expressions of pfl $\mathrm{AB}$ and ace $\mathrm{AB}$ genes are affected under the low DO conditions (Böck et al. 2008; Lynch and Lin 1996), but also affects cell viability at the switch to the anaerobic production phase in the current study.

During the anaerobic phase, the specific succinate production rate drops as succinate accumulates (Figs. 1 and 8 ) as cell viability decrease (Fig. 2). E. coli subjected to osmotic stress respond by accumulating compatible solutes, such as glycine betaine, proline, and trehalose (Larsen et al. 1987). Among these osmoprotectants, glycine betaine offers the highest osmotolerance in $E$. coli (Underwood et al. 2004) and other cells (Haussinger and Lang 1991; Robinson et al. 1986). E. coli can only accumulate glycine betaine or proline if supplemented in the medium or by a two-step oxidation of externally provided choline. Andersson et al. (2009) reported that addition of glycine betaine to the cultures of $E$. coli AFP184 resulted in an increased aerobic growth rate and anaerobic glucose consumption rate but decreased succinic acid yield. In contrast, studies with $E$. coli engineered for lactic acid production has shown that betaine greatly increased the volumetric lactic acid productivity (Zhou et al. 2006). In the present investigation, cell aerobic growth rate, glucose consumption, and succinate production were not affected by the addition of betaine. We are currently pursuing other approaches such as strain evolvolution under succinate pressure to improve cell viability during succinate production process.

The $\mathrm{pH}$ was not controlled in most of the fermentations during the aerobic phase in the current study. Instead, the $\mathrm{pH}$ was used as an indicator of glucose consumption to determine glucose feeding times. Less osmotic stress was introduced as an obvious benefit by allowing the $\mathrm{pH}$ to freely change as the cell produced or utilized organic acids during the A-I and A-II phases. However, we also observed that the succinate yield will be much lower if the $\mathrm{pH}$ dropped to below 5.5 during the A-I or A-II phases. Therefore, the amount of glucose feeding was carefully set at about $2 \mathrm{~g} / \mathrm{L}$ each time so that acid production can be controlled at an appropriate level.

Controlling the culture $\mathrm{pH}$ at 7.0 was expected to give better cell growth and therefore improve overall succinate productivity during the anaerobic phase. However, both the succinate yield and specific succinate production rate were significantly lower than those in the experiments without $\mathrm{pH}$ control. Osmotic stress could be introduced since the $\mathrm{pH}$ was maintained by adding acid and base during organic acid production and absorption periods. Besides, the low DO incubation, which is recognized as a critical phase in the current study, was disturbed by the $\mathrm{pH}$ control since the feeding of acid and base not only changes the medium composition, but also may stress the cell and we observed DO fluctuations during the experiments. We noticed higher pyruvate accumulation in the $\mathrm{pH}$ controlled experiments (data not shown), which suggests that the controlled $\mathrm{pH}$ may affect the fine tuning of gene expression related to pyruvate metabolism. Therefore, confining the $\mathrm{pH}$ change range with careful glucose feeding and letting the $\mathrm{pH}$ freely change according to cell metabolism seems the best strategy for the current system so far.

Formate is one of the major side products during the fermentation of the current strain (Figs. 1 and 8). Besides its inhibition to cell growth, formate can also serve as a potential reducing power provider if dehydrated through formate dehydrogenase (Berrios-Rivera et al. 2002). Data 
from other experiments we have conducted suggested that the $E$. coli native FDH may be activated at about $\mathrm{pH} 6.5$. Therefore, two experiments were conducted by controlling the $\mathrm{pH}$ at 6.2 and 6.3, respectively, during the aerobic phase (Fig. 6). However, such conditions significantly reduced the succinate yield in these cultures. The low $\mathrm{pH}$ or the $\mathrm{pH}$ control reagents may have disturbed the culture conditions.

The cell viability loss during the anaerobic phase is not favorable for the succinate production (Figs. 1 and 8). The high osmotic pressure caused by succinate production and addition of neutralization reagents could be a major stress for the cell. Therefore, further strain engineering is still required for the most economical production of succinate. Since it is observed that the sizes of a large portion of cells increased during the succinate production phase (Fig. 2), one would speculate that the cell may suffer from inefficient excretion of the organic acid produced from glucose. Limiting glucose uptake rate by modifying the phosphotransferase system may help on maintaining cell viability, but it will also reduce the cell productivity, which is not preferred for succinate production. Modifying or introducing a robust heterologous succinate transport system may be helpful for future solution of the cell viability issue. Besides, the Pyc expressed using the plasmid system may also result in a high cell burden during the growth phase, which reduces cell density and viability at the switch to the anaerobic phase. Incorporating the $p y c$ gene expression system on the chromosome may also help cells grow better under the stressful conditions of succinate production.

In summary, the aerobic phase culture conditions significantly affected the metabolic behavior of SBS550MG (pHL413) and therefore influence the anaerobic succinate production. To briefly conclude, we focused on the study of key operating conditions of the aerobic growth phase. It was found that $2-5 \mathrm{~h}$ of low DO culture during the aerobic phase improves cell productivity, and that $\mathrm{pH}$ control during the aerobic phase was not favorable for the fine tuning of enzymatic system of cells. It has been noticed that the cell viability decreased significantly after $20 \mathrm{~h}$ of succinate production, and supplying LB medium and betaine did not improve the cell activity. A higher succinate titer $(537.8 \mathrm{mM})$ using the current metabolically engineered $E$. coli strain was achieved as compared to previous work (Sanchez et al. 2005; Martinez et al. 2010), and this titer apparently can still be improved by addressing the cell viability issue.

Acknowledgment The authors appreciate Mary Harrison for her creative and reliable support on the lab management and technical advice. We also thank Irene Martinez for her constructive discussions and suggestions on the experiments. This work was supported by funding from ROQUETTE FRERES, France.

\section{References}

Andersson C, Helmerius J, Hodge D, Berglund KA, Rova U (2009) Inhibition of succinic acid production in metabolically engineered Escherichia coli by neutralizing agent, organic acids, and osmolarity. Biotechnol Prog 25(1):116-123

Baneyx F (1999) Recombinant protein expression in Escherichia coli. Curr Opin Biotechnol 10(5):411-421

Berrios-Rivera SJ, Bennett GN, San KY (2002) The effect of increasing NADH availability on the redistribution of metabolic fluxes in Escherichia coli chemostat cultures. Metab Eng 4(3):230-237

Böck A, Curtiss R III, Kaper JB, Karp PD, Neidhardt FC, Nyström T, Slauch JM, Squires CL, Ussery D (2008) EcoSal-Escherichia coli and Salmonella: cellular and molecular biology. ASM Press, Washington DC

Cox SJ, Shalel Levanon S, Sanchez A, Lin H, Peercy B, Bennett GN, San KY (2006) Development of a metabolic network design and optimization framework incorporating implementation constraints: a succinate production case study. Metab Eng 8:46-57

Cozzone AJ (1998) Regulation of acetate metabolism by protein phosphorylation in enteric bacteria. Annu Rev Microbiol 52:127-164

Haacke A, Fendrich G, Ramage P, Geiser M (2009) Chaperone overexpression in Escherichia coli: apparent increased yields of soluble recombinant protein kinases are due mainly to soluble aggregates. Protein Expr Purif 64(2):185-193

Haussinger D, Lang F (1991) Cell volume in the regulation of hepatic function: a mechanism for metabolic control. Biochimica Et Biophysica Acta 1071(4):331-50

Hong SH, Lee SY (2002) Importance of redox balance on the production of succinic acid by metabolically engineered Escherichia coli. Appl Microbiol Biotechnol 58(3):286-290

Jantama K, Haupt MJ, Svoronos SA, Zhang X, Moore JC, Shanmugam KT, Ingram LO (2008) Combining metabolic engineering and metabolic evolution to develop nonrecombinant strains of Escherichia coli $\mathrm{C}$ that produce succinate and malate. Biotechnol Bioeng 99(5):1140-1153

Larsen PI, Sydnes LK, Landfald B, Strom AR (1987) Osmoregulation in Escherichia coli by accumulation of organic osmolytes: betaines, glutamic acid, and trehalose. Arch Microbiol 147:1-7

Lee PC, Lee WG, Lee SY, Chang HN (2001) Succinic acid production with reduced by-product formation in the fermentation of Anaerobiospirillum succiniciproducens using glycerol as a carbon source. Biotechnol Bioeng 72(1):41-48

Lee PC, Lee SY, Hong SH, Chang HN, Park SC (2003) Biological conversion of wood hydrolysate to succinic acid by Anaerobiospirillum succiniciproducens. Biotechnol Lett 25(2):111-114

Lee SJ, Song H, Lee SY (2006) Genome-based metabolic engineering of Mannheimia succiniciproducens for succinic acid production. Appl Environ Microbiol 72(3):1939-1948

Lynch AS, Lin EC (1996) Transcriptional contorl mediated by the ArcA two-component response regulator protein of Eschericha coli: characterization of DNA binding at target promoters. J Bacteriol 178:6238-6249

Martinez I, Bennett GN, San KY (2010) Metabolic impact of the level of aeration during cell growth on anaerobic succinate production by an engineered Escherichia coli strain. Metab Eng 12:499-509

McKinlay JB, Zeikus JG, Vieille C (2005) Insights into Actinobacillus succinogenes fermentative metabolism in a chemically defined growth medium. Appl Environ Microbiol 71(11):6651-6656

Oh IJ, Lee HW, Park CH, Lee SY, Lee J (2008) Succinic acid production by continuous fermentation process using Mannheimia succiniciproducens LPK7. J Microbiol Biotechnol 18 (5):908-912 
Otero JM, Olsson L, Nielsen J (2007) Metabolic engineering of Saccharomyces cerevisiae microbial cell factories for succinic acid production. J Biotechnol 131(2):S205-S205

Portle S, Causey TB, Wolf K, Bennett GN, San KY, Mantzaris N (2007) Cell population heterogeneity in expression of a geneswitching network with fluorescent markers of different halflives. J Biotechnol 128(2):362-375

Robinson AC, Burgess BK, Dean DR (1986) Activity, reconstitution, and accumulation of nitrogenase components in Azotobacter vinelandii mutant strains containing defined deletions within the nitrogenase structural gene cluster. J Bacteriol 166(1):180-186

Sanchez AM, Bennett GN, San KY (2005) Novel pathway engineering design of the anaerobic central metabolic pathway in Escherichia coli to increase succinate yield and productivity. Metab Eng 7(3):229-239

Sanchez AM, Bennett GN, San KY (2006) Batch culture characterization and metabolic flux analysis of succinate-producing Escherichia coli strains. Metab Eng 8:209-226

Underwood SA, Buszko MT, Shanmugam KT, Ingram LO (2004) Lack of protective osmolytes limits final cell density and volumetric productivity of ethanologenic Escherichia coli KO11 during xylose fermentation. Appl Environ Microbiol 70:27342740

Vemuri GN, Eiteman MA, Altman E (2002) Succinate production in dual-phase Escherichia coli fermentations depends on the time of transition from aerobic to anaerobic conditions. J Ind Microbiol Biotechnol 28(6):325-332

Walsh K, Koshland DE (1985) Branch point control by the phosphorylation state of isocitrate dehydrogenase. A quantitative examination of fluxes during a regulatory transition. J Biol Chem 260(14):8430-8437

Werpy T, Petersen G (2004) Top value-added chemicals from biomass -volume I. US Department of Energy, Washington, DC

Yang YT, Bennett GN, San KY (1999) Effect of inactivation of nuo and ackA-pta on redistribution of metabolic fluxes in Escherichia coli. Biotechnol Bioeng 65(3):291-297

Zhou S, Grabar TB, Shanmugam KT, Ingram LO (2006) Betaine tripled the volumetric productivity of D (-)-lactate by Escherichia coli strain SZ132 in mineral salts medium. Biotechnol Lett 28:671-676 